\title{
Holographic Phonons
}

\author{
Lasma Alberte* \\ Abdus Salam International Centre for Theoretical Physics (ICTP), Strada Costiera 11, 34151 Trieste, Italy \\ Martin Ammon ${ }^{\dagger}$ and Amadeo Jiménez-Alba \\ Theoretisch-Physikalisches Institut, Friedrich-Schiller-Universität Jena, Max-Wien-Platz 1, D-07743 Jena, Germany \\ Matteo Baggioli ${ }^{\S}$ \\ Crete Center for Theoretical Physics, Institute for Theoretical and Computational Physics Department of Physics, \\ University of Crete, 71003 Heraklion, Greece \\ Oriol Pujolàs\| \\ Institut de Física d'Altes Energies (IFAE), \\ The Barcelona Institute of Science and Technology (BIST) Campus UAB, 08193 Bellaterra, Barcelona
}

(Received 7 December 2017; revised manuscript received 6 February 2018; published 27 April 2018)

We present a class of holographic massive gravity models that realize a spontaneous breaking of translational symmetry - they exhibit transverse phonon modes whose speed relates to the elastic shear modulus according to elasticity theory. Massive gravity theories thus emerge as versatile and convenient theories to model generic types of translational symmetry breaking: explicit, spontaneous, and a mixture of both. The nature of the breaking is encoded in the radial dependence of the graviton mass. As an application of the model, we compute the temperature dependence of the shear modulus and find that it features a glasslike melting transition.

DOI: 10.1103/PhysRevLett.120.171602

Introduction.-In the last decade the gauge/gravity duality has proven to be an efficient tool to tackle condensed matter questions in the context of strongly coupled physics [1-3]. Despite the various directions and applications pursued, a fundamental piece of the condensed matter phenomenology is still missing in the holographic puzzle: a concrete, simple, and clear realization of phonons with standard properties as dictated by elasticity theory; see e.g. Ref. [4]. With the present Letter we shall rectify this deficiency by presenting a class of simple holographic models featuring transverse phonons and elastic properties.

Recently, there has been significant progress towards including translational symmetry breaking, momentum dissipation, and their consequences on transport in the context of holography [5,6] (see Ref. [1] for references). Within that framework, Massive gravity (MG) stands out as a convenient and flexible gravity dual where the momentum relaxation time is set by the graviton mass $\tau_{\text {rel }}^{-1} \sim m_{g}^{2}$ [7-9]. The question regarding the nature of the translational symmetry breaking, i.e., whether it occurs in a spontaneous

Published by the American Physical Society under the terms of the Creative Commons Attribution 4.0 International license. Further distribution of this work must maintain attribution to the author(s) and the published article's title, journal citation, and DOI. Funded by SCOAP ${ }^{3}$. or explicit manner, is, however, subtle. According to the holographic dictionary, the answer lies in the asymptotic UV behavior of the bulk fields breaking the translational invariance [10]. In the case of massive gravity this relates to the radial dependence of the graviton mass, which was shown to admit a broad range of possible profiles $m_{g}(u)$ compatible with theoretical consistency $[11,12]$.

A first evidence confirming this logic was presented in Ref. [13], where gapped transverse phonons were identified, with the size of the gap being directly related to the asymptotic behavior of the graviton mass. This suggests a clear way to realize gapless phonons by ensuring a rapid enough decay of $m_{g}(u)$ towards the boundary.

In this Letter we consider a subclass of the holographic MG models introduced in Refs. [11,12] exhibiting such behavior. We then demonstrate explicitly how it can attain a spontaneous symmetry breaking (SSB) of translations and provide a realization of massless phonons, i.e., the corresponding Goldstone bosons. The resulting gapless modes show properties identical to the transverse phonons in solids. In particular, we find that their speed of propagation is in perfect agreement with the expectations from elasticity theory [14]. To the best of our knowledge this is the first time that transverse phonons are realized within holography, with a sharp and clear relation to the elastic moduli as dictated by standard elasticity theory. Previous works with partial success in this direction are listed in Ref. [15]. 
Despite the fact that the physics of phonon excitations and elasticity in weakly coupled solids is well known, their holographic realization has remained absent for more than a decade. We believe that the present work will clarify how the phonons can be encoded in the holographic models. Beyond the AdS/CFT dictionary, this will certainly contribute to a better understanding of the role of phonons, and more generally of spontaneous and explicit breaking of translational invariance, especially in quantum critical or in strongly coupled materials. In addition, this is of relevance for metal-insulator transitions and in disordered materials, where the gapping or pinning of phonons plays a crucial role. Pinning and its effects on transport phenomena can be readily modeled using the holographic methods presented in this work. Finally, let us remark that these models allow for a concrete derivation of the hydrodynamics of certain ordered phases of matter and their related viscoelastic transport coefficients at strong coupling.

Holographic setup.-We consider generic solid holographic massive gravity models $[11,12]$ :

$$
S=M_{P}^{2} \int d^{4} x \sqrt{-g}\left(\frac{R}{2}+\frac{3}{\ell^{2}}-m^{2} V(X)-\frac{1}{4} F^{2}\right),
$$

with $X \equiv \frac{1}{2} g^{\mu \nu} \partial_{\mu} \phi^{I} \partial_{\nu} \phi^{I}$ and $F^{2}=F_{\mu \nu} F^{\mu \nu}$. We study 4D AdS black brane geometries of the form

$$
d s^{2}=\frac{\ell^{2}}{u^{2}}\left[\frac{d u^{2}}{f(u)}-f(u) d t^{2}+d x^{2}+d y^{2}\right],
$$

where $u \in\left[0, u_{h}\right]$ is the radial holographic direction spanning from the boundary to the horizon, defined through $f\left(u_{h}\right)=0$, and $\ell$ is the AdS radius.

The $\phi^{I}$ scalars are the Stückelberg fields admitting a radially constant profile $\phi^{I}=x^{I}$ with $I=x, y$. This is an exact solution of the system due to the shift symmetry. In the dual picture these fields represent scalar operators breaking the translational invariance because of the explicit dependence on the spatial coordinates. In this Letter we shall consider benchmarks models of the type

$$
V(X)=X^{n} .
$$

These are referred to as massive gravity theories because, among other reasons, the metric perturbations acquire a mass term given by $m_{g}^{2}(u)=2 m^{2} X V^{\prime}$ with the background value for $X=u^{2} / \ell^{2}$. The absence of ghost and gradient instabilities enforces the conditions $V^{\prime}>0$ and $c_{\text {(bulk) }}^{2}=1+$ $X V^{\prime \prime} / V^{\prime}>0$ which constrain the power to satisfy $n>0$ [11].

In the following we assume standard quantization. This means that the near-boundary leading mode of the Stückelberg fields, $\phi_{(l)}^{I}$, sets the source for the dual operator $\mathcal{O}_{I}$ breaking the translational invariance. The expectation value $\left\langle O_{I}\right\rangle$ is in turn set by the subleading mode $\phi_{(s)}^{I}$.

For potentials of the type (3) the asymptotic expansion of the Stuickelberg scalars close to the UV boundary at $u=0$ is given by

$$
\phi^{I}\left(x^{\mu}\right)=\phi_{(0)}^{I}\left(t, x^{i}\right)+\phi_{(1)}^{I}\left(t, x^{i}\right) u^{5-2 n}+\ldots,
$$

where $\phi_{(0)}^{I}\left(t, x^{i}\right)=x^{I} \neq 0$ on the bulk solution. Depending on the value of $n$ one can then distinguish two cases. If $n<5 / 2$ then $\phi_{(0)}^{I}$ is the leading term in the near-boundary expansion and corresponds to the source, i.e., $\phi_{(l)}^{I}=x^{I}$. As a consequence, the dual QFT contains an explicit breaking term which gives rise to a finite relaxation time $\tau_{\text {rel }}^{-1} \sim m_{g}^{2}$ for the momentum operator [8]. This is the case for all the potentials $V(X)$ that have so far been considered in the literature [11-13].

The main observation is that if instead one considers a potential of the form $V(X)=X^{n}$ with a sufficiently large $n>5 / 2$, the mode $\phi_{(0)}^{I}$ becomes subleading in the boundary expansion (4). Hence, for $n>5 / 2$ the solution $\phi^{I}=x^{I}$ for the scalar bulk fields gives rise to an expectation value $\left\langle\mathcal{O}_{I}\right\rangle \neq 0$ for its dual operator while its source vanishes, leading to the SSB pattern [24]. Intuitively such a condition corresponds to demanding that the radially dependent graviton mass $m_{g}(u)$ is large at the horizon and quickly vanishes at the boundary, as already suggested in Refs. [11,13].

Let us now focus on an important feature of the benchmark models (3). For $n \neq 1$-our main focus in this workthe kinetic term for the Stückelberg fields $\phi^{I}$ is noncanonical: they do not have a quadratic action. This implies that their quantization is at best nonstandard, and thus the theory is strongly coupled around the trivial solution $\phi^{I}=$ const. For this reason, the interpretation of this classical solution as a valid quantum vacuum is quite dubious.

Instead, around the nontrivial solution $\phi^{I}=x^{I}$ the Stuickelberg fields do acquire a standard quadratic kinetic term, at least in part of the geometry. To illustrate this, it suffices to consider the Stuickelberg fields in the transverse sector. Separating them into background and perturbations as $\phi^{I}=x^{I}+\pi^{I}$, with $\partial_{i} \pi^{i}=0$, one can easily expand the Lagrangian in powers of $\pi$ 's to find

$$
-M_{P}^{2} m^{2} \int d^{4} x \sqrt{-g}\left\{V^{\prime}(\bar{X}) X_{\pi}+\frac{1}{2} V^{\prime \prime}(\bar{X}) X_{\pi}^{2}+\ldots\right\},
$$

where $\bar{X}$ denotes the background value, $u^{2} / \ell^{2}$, and $X_{\pi} \equiv \frac{1}{2} g^{\mu \nu} \partial_{\mu} \pi^{I} \partial_{\nu} \pi^{I}$. One can estimate the strong coupling scale in this sector by going to canonical normalization $\pi_{c}=M_{P} m \sqrt{V^{\prime}} \pi$ and finding the scale suppressing the dimension-8 operator $\left(\partial \pi_{c}\right)^{4} / \Lambda(u)^{4}$. The resulting strong coupling scale is radial dependent and is given by $\Lambda(u)^{4} \sim\left(V^{\prime 2} / V^{\prime \prime}\right) M_{P}^{2} m^{2}$. For our benchmark models (3) with generic $n$ this gives

$$
\Lambda(u) \sim\left(m M_{P}\right)^{1 / 2}\left(\frac{u}{\ell}\right)^{n / 2} .
$$




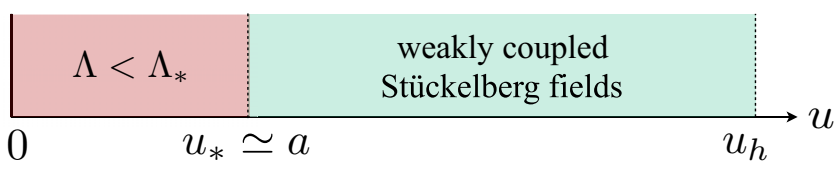

FIG. 1. A sketch of the validity of the EFT in the bulk and its dual interpretation.

Thus, for a fixed $m^{2}$ the energy scale above which the transverse field perturbations $\pi^{I}$ become strongly coupled varies with the radial distance $u$.

Since $\Lambda(u)$ asymptotically vanishes towards the AdS boundary located at $u=0$, then in practice our bulk EFT is tractable only down to a certain radius $u_{*}$ as depicted in Fig. 1. In the dual CFT the scale $u_{*}$ clearly corresponds to some UV cutoff. This is a very welcome feature since we do expect the strength of phonon self-interactions to increase towards high energies, as in weakly coupled materials; see, e.g., Ref. [26]. It is therefore tempting to relate the scale $u_{*}$ with the lattice spacing scale $a$, setting an upper cutoff to the phonons frequency. In physical terms this cutoff indicates that the phononic vibrational modes cannot be excited above the so-called Debye temperature [27]. Therefore, it is neither surprising nor problematic to have a UV cutoff in our gravitational theory; on the contrary, it is an important physical property making these models more realistic. How small $u_{*}$ is depends on how small we can tolerate the Stückelberg strong coupling scale $\Lambda_{*}$ anywhere in the bulk. Notice that this is a new parameter in the model, independent from the parameters appearing in Eq. (1) [28].

There are two basic and obvious constraints in choosing $\Lambda_{*}$. First, $\Lambda_{*}$ must be bigger than the typical gradients, that is, $\Lambda_{*} \gg 1 / \ell$. Second, in order to still be able to read off the holographic correlators from the decay modes of the bulk fields, we also need the UV cutoff $u_{*}$ to be close to the AdS boundary. In other words, we must ensure that the ratio $u_{*} / \ell$ is sufficiently small.

Thus, we can write $\Lambda_{*}=\left(m M_{P}\right)^{1 / 2}\left(u_{*} / \ell\right)^{n / 2}$ and think of $u_{*} / \ell$ as a fixed small number. Requiring that $\Lambda_{*} \ell \gtrsim 1$ then gives

$$
M_{P} \ell \gtrsim \frac{1}{m \ell}\left(\frac{u_{*}}{\ell}\right)^{-n} .
$$

Note that $M_{P} \ell$ has to be large for the employed semiclassical treatment of the gravitational side to be valid. Hence, for any given small $u_{*} / \ell$ and any $n$, we can satisfy the two constraints with $m \ell$ of order one. Once these conditions are met, then these constructions allow us to model in a controlled way the physics of phonons in critical and conformal solids.

Results.-Phonons and elasticity: In solids translational invariance is spontaneously broken. The corresponding Goldstone bosons - the phonons-play a crucial role in the description of the low energy physics and the elastic properties of the materials. Their dynamics can be entirely captured via effective field theory methods [26,29]. Depending on the direction of propagation with respect to the deformation of the medium they can be classified into longitudinal and transverse phonons; in this Letter we shall focus on the latter. The presence of propagating transverse phonons, also called the shear sound, is a characteristic property of solids and provides a clear physical distinction from fluids.

The dispersion relation for the transverse phonons takes the simple form [4]

$$
\omega=c_{T} k-i D k^{2},
$$

where $c_{T}$ is the speed of propagation and $D$ is the momentum diffusion constant, proportional to the finite viscosity $\eta$ of the medium. In the absence of explicit breaking, neither mass gap nor damping is present; i.e., at low $k$ the real part of the frequency depends linearly on $k$, as shown in Eq. (6) [30]. Moreover, note that in the absence of translational symmetry breaking the mode is purely diffusive, with $c_{T}=0$. In relativistic systems, the velocity is set to be $[4,32-34]$

$$
c_{T}^{2}=\frac{G}{\chi_{P P}},
$$

where $G$ is the shear elastic modulus and $\chi_{P P}$ is the momentum susceptibility. Both of these quantities can be extracted via the Kubo formulas from the $T_{x y}$ (shear stress) and $T_{t x}$ (momentum) retarded correlators as follows:

$$
\left.G \equiv \mathcal{G}_{T_{x y} T_{x y}}^{(\mathrm{R})}\right|_{\omega, k=0},\left.\quad \chi_{P P} \equiv \mathcal{G}_{T_{t x} T_{t x}}^{(\mathrm{R})}\right|_{\omega, k=0} .
$$

The momentum susceptibility coincides with [1],

$$
\chi_{P P}=T_{00}+T_{x x}=\epsilon+\mathcal{P}=\frac{3}{2} \epsilon,
$$

where $\epsilon$ is the energy density and $\mathcal{P}$ is the mechanical pressure [35].

In order to confirm the presence of transverse phonons and verify Eq. (7), we find the spectrum of the quasinormal modes (QNMs) of the system in the transverse sector. Here we have done it explicitly for potentials of the type $V(X)=$ $X^{n}$ for several values of $n \in[3-8]$ including also noninteger values [37]. For concreteness, some of the following plots only show specific realizations; nevertheless, the qualitative conclusions drawn from the data are the same in all cases.

In Fig. 2 we show the spectrum of QNMs at zero momentum and different temperatures for $n=5$. We find a QNM located at zero frequency, corresponding to a gapless quasiparticle, the putative phonon in our holographic model. Moreover, we note that for any temperature the next QNM is already highly damped.

Next, we analyze the QNM spectrum at finite momentum $k$. In Fig. 3 we show the behavior of both its real and 


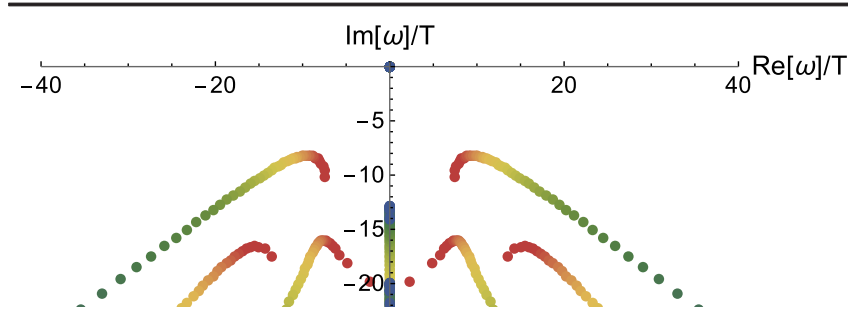

FIG. 2. The quasinormal spectrum for $V(X)=X^{5}$ at zero momentum for $T / m \in[0.01,1.05]$ (blue-red).

imaginary parts. As evident from Fig. 3, this quasinormal mode satisfies the expected dispersion relation of Eq. (6) [38]. Hence it is neither attenuated nor gapped. We note that the speed $c_{T}$ decreases with increasing temperature (see Fig. 4) while the diffusion constant $D$ increases with $T / m$. In particular, at $T / m=0$ the diffusion constant and the viscosity vanish $D=\eta=0$ (see also Refs. [36,39]) and the elastic modulus $G$ is maximal. In turn, at $T / m \gg 1$ the viscosity is maximal and the elastic modulus is zero. In other words, the physics interpolates from a solid behavior at zero temperature to a fluid behavior at high temperatures in a continuous way, exhibiting viscoelastic features in the intermediate temperature range. This feature is qualitatively similar to a glassy transition typical to viscoelastic materials [40-42], as shown in the inset of Fig. 4 and explained in more detail in the Supplemental Material [43]. Although it is to be expected that the system interpolates between a fluid and a "solid," it is worth noting that the transition is smooth.

Crucially, as shown in Fig. 4, the sound speed of the transverse phonons is in perfect agreement with the expectations from the elastic theory given in Eq. (7). We note that the value of the velocity in the zero-temperature limit is always subluminal, but not universal. Moreover, we find that for $n \geq 3$ the sound speed satisfies the bound $c_{T}^{2} \leq 1 / 2$ arising for conformal solids [23].

Conductivity and viscosity.-By introducing a finite charge density $\rho$ we are able to analyze also the electric optical conductivity $\sigma(\omega)$ of our system. In the presence of only SSB its low frequency expansion is expected to be given by [1]

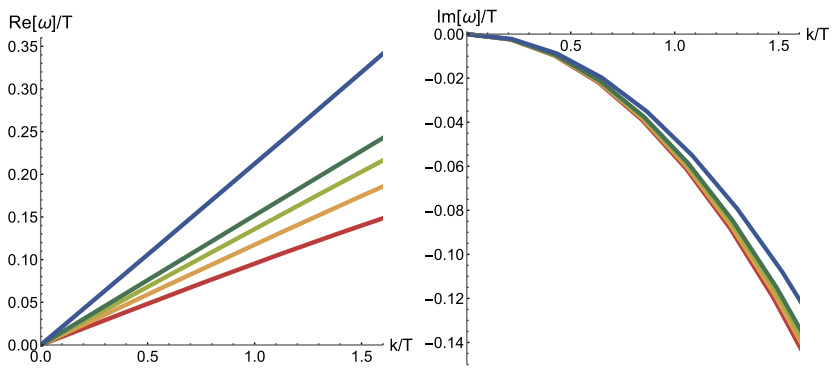

FIG. 3. Real and imaginary parts of the frequency of the lowest QNM for $V(X)=X^{5}$ for $T / m \in[0.7-1.7]$ (blue-red).

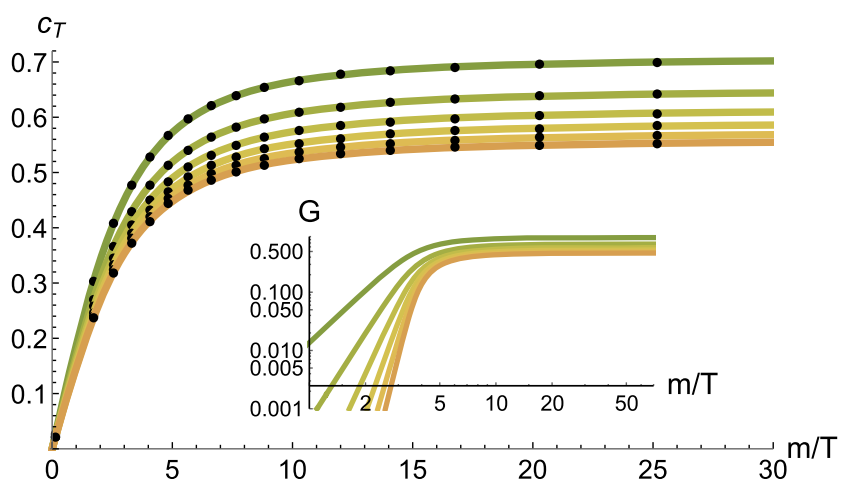

FIG. 4. Comparison of the velocity extracted from the QNMs (black dots) and the velocity computed from the elasticity (solid lines) for $n \in[3-8]$ (green-orange). Inset: The dependence of the shear elastic modulus on $m / T$ in $\log$-log scale. The fall-off at large temperatures $G \sim m^{2} T^{3-2 n}$ is evident. See the Supplemental Material [43].

$$
\sigma(\omega)=\sigma_{Q}+\frac{\rho^{2}}{\chi_{P P}}\left(\delta(\omega)+\frac{i}{\omega}\right)
$$

where $\sigma_{Q}$ is the so-called incoherent conductivity (see Refs. [31,46]). Notice that because of the absence of a finite momentum relaxation time $\tau_{\text {rel }}$, or, equivalently, of an explicit breaking mechanism, the dc conductivity is infinite.

We have computed the optical conductivity of our class of models via the Kubo formula:

$$
\sigma(\omega)=\left.\frac{1}{i \omega} \mathcal{G}_{J J}^{(\mathrm{R})}(\omega)\right|_{k=0} .
$$

As shown in Fig. 5, the low frequency behavior of the conductivity agrees with the form presented in Eq. (10); also the Drude weight is in perfect agreement with the hydrodynamics expectations. This allows us to confidently claim that our setup Eq. (1) does indeed represent a dual of the SSB of translational invariance, together with all its physical manifestations.

Finally, let us comment on the momentum diffusion constant $D$ appearing in the dispersion relation (6) and related to the hydrodynamical viscosity $\eta$ by $D=\eta / \chi_{P P}$.

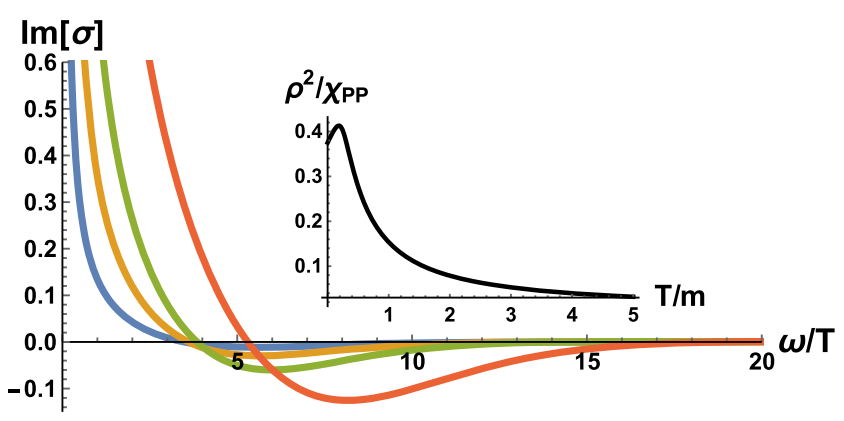

FIG. 5. Imaginary part of $\sigma(\omega)$ for $V(X)=X^{5}$ at $\mu=1$ and different $T / m=1,0.6,0.4,0.2$. In the inset the Drude weight, $\rho^{2} / \chi_{P P}$, is shown. 
We find that $\eta$ does not agree with the value extracted via the Kubo formula:

$$
\eta^{*}=-\lim _{\omega \rightarrow 0} \frac{1}{\omega} \operatorname{Im} \mathcal{G}_{T_{x y} T_{x y}}^{(\mathrm{R})}(k=0) .
$$

This was already noticed for the explicit breaking case with $n=1$ in Refs. [47,48]. This point has been further elaborated recently in [49]. Here we confirm this disagreement between the two viscosities for generic values of $n$.

Conclusions. - We present a simple holographic gravity dual for phonons in strongly coupled materials, whose properties are in perfect agreement with elasticity theory. Our results open a new window for the study of strongly coupled solids via the AdS/CFT methods. In the process, we also sharpen and quantify the connection between elastic theory and massive gravity $[7,11,12,50]$.

The future possibilities are diverse. One direction of clear interest is to compute and characterize the viscoelastic response of these models in more detail. This will clarify the connection with the known glassy melting transitions [40-42]. In this regard it seems relevant to study the response under time-dependent stresses as it could shed light on further signatures typical for amorphous and viscoelastic materials like slow relaxation and aging [51] (see, e.g., Ref. [52] for previous works).

In addition, it would be compelling to elucidate any possible relation to the so-called quantum critical elasticity $[53,54]$.

Finally, these methods can be used to model the optical transport properties of the strange and bad metals in terms of the interplay between the explicit and spontaneous breaking of translations $[49,55]$.

We thank A. Amoretti, D. Arean, A. Beekman, A. Esposito, S. Garcia-Saenz, S. Grozdanov, S. Hartnoll, K. Y. Kim, A. Krikun, J. Leiber, D. Musso, A. Nicolis, N. Obers, C. Pantelidou, R. Penco, N. Poovuttikul, S. J. Sin, J. Zaanen and, in particular, B. Gouteraux for useful discussions and comments about this work and the topics considered. We are grateful to J. Zaanen for reading a preliminary version of this letter and providing insightful comments. M. B. is supported in part by the Advanced ERC Grant SM-grav, No. 669288. A. J. acknowledges financial support by Deutsche Forschungsgemeinschaft (DFG) GRK 1523/2. O. P. acknowledges support by the Spanish Ministry MEC under Grant No. FPA2014-55613-P and the Severo Ochoa excellence program of MINECO (Grant No. SO2012-0234, SEV-2016-0588), as well as by the Generalitat de Catalunya under Grant No. 2014-SGR-1450. M. B. would like to thank the Nordic Institute for Theoretical Physics (NORDITA) and the organizers of the "Many-Body Quantum Chaos, Bad Metals and Holography" workshop for the hospitality during the completion of this work. M. B. would also like to "thank" the University General Hospital of Heraklion Pagni for the long hospitality during the completion of this Letter.
Note added.-Recently, we became aware of upcoming works discussing similar issues $[56,57,58]$.

\author{
*lalberte@ictp.it \\ †martin.ammon@uni-jena.de \\ †amadeo.jimenez.alba@uni-jena.de \\ \$mbaggioli@physics.uoc.gr \\ "pujolas@ifae.es
}

[1] S. A. Hartnoll, A. Lucas, and S. Sachdev, arXiv:1612.07324.

[2] M. Ammon and J. Erdmenger, Gauge/Gravity Duality (Cambridge University Press, Cambridge, England, 2015).

[3] J. Zaanen, Y. Liu, Y. Sun, and K. Schalm, Holographic Duality in Condensed Matter Physics (Cambridge University Press, Cambridge, England, 2015).

[4] P. M. Chaikin and T. C. Lubensky, Principles of Condensed Matter Physics (Cambridge University Press, Cambridge, England, 1995).

[5] S. A. Hartnoll and D. M. Hofman, Phys. Rev. Lett. 108, 241601 (2012)

[6] G. T. Horowitz, J. E. Santos, and D. Tong, J. High Energy Phys. 07 (2012) 168.

[7] D. Vegh, arXiv:1301.0537.

[8] R. A. Davison, Phys. Rev. D 88, 086003 (2013).

[9] M. Blake and D. Tong, Phys. Rev. D 88, 106004 (2013).

[10] I. R. Klebanov and E. Witten, Nucl. Phys. B556, 89 (1999).

[11] M. Baggioli and O. Pujolàs, Phys. Rev. Lett. 114, 251602 (2015).

[12] L. Alberte, M. Baggioli, A. Khmelnitsky, and O. Pujolas, J. High Energy Phys. 02 (2016) 114.

[13] L. Alberte, M. Ammon, M. Baggioli, A. Jiménez, and O. Pujolàs, J. High Energy Phys. 01 (2018) 129.

[14] L. D. Landau and E. M. Lifshitz, Course of Theoretical Physics, Vol. 7, Theory of Elasticity (Pergamon Press, New York, 1970).

[15] The SSB of translations has been previously realized in holography in Refs. [16-21] as the dual of charge density waves states [22], where the Goldstone boson is identified with the so-called sliding mode. Another realization of gapless transverse phonon modes was made in Ref. [23], using a model with more dynamical ingredients - even though a direct comparison to the elastic moduli is lacking. Finally, let us also mention that a gapped transverse phonon was realized in Ref. [13] yet with a mass gap that is parametrically small and therefore close to the exact SSB limit.

[16] S. Nakamura, H. Ooguri, and C.-S. Park, Phys. Rev. D 81, 044018 (2010).

[17] A. Donos and J. P. Gauntlett, Phys. Rev. D 86, 064010 (2012).

[18] A. Donos and J. P. Gauntlett, Phys. Rev. D 87, 126008 (2013).

[19] A. Donos and J. P. Gauntlett, J. High Energy Phys. 08 (2011) 140.

[20] T. Andrade, M. Baggioli, A. Krikun, and N. Poovuttikul, J. High Energy Phys. 02 (2018) 085.

[21] N. Jokela, M. Jarvinen, and M. Lippert, Phys. Rev. D 96, 106017 (2017).

[22] G. Grüner, Rev. Mod. Phys. 60, 1129 (1988).

[23] A. Esposito, S. Garcia-Saenz, A. Nicolis, and R. Penco, J. High Energy Phys. 12 (2017) 113.

[24] We thank Blaise Gouteraux for suggesting this interpretation. See also Ref. [25] for a detailed analysis on the nature 
of the translational symmetry breaking patterns in QFT and holography.

[25] A. Amoretti, D. Areán, R. Argurio, D. Musso, and L. A. Pando Zayas, J. High Energy Phys. 05 (2017) 051.

[26] H. Leutwyler, Helv. Phys. Acta 70, 275 (1997).

[27] J. Garai, Astropart. Phys. 29, 1 (2008).

[28] This new parameter does not appear in models with a standard kinetic term near $X=0$, i.e., $V(X)=X+X^{2}+\ldots$ because there $\Lambda(u)$ does not asymptote to zero.

[29] A. Nicolis, R. Penco, F. Piazza, and R. Rattazzi, J. High Energy Phys. 06 (2015) 155.

[30] This is opposed to the case of mass potentials $V(X)=X^{n}$ with $n<5 / 2$, studied in Refs. [8,13,31].

[31] R. A. Davison and B. Goutéraux, J. High Energy Phys. 01 (2015) 039.

[32] P. C. Martin, O. Parodi, and P. S. Pershan, Phys. Rev. A 6, 2401 (1972).

[33] A. Zippelius, B. I. Halperin, and D. R. Nelson, Phys. Rev. B 22, 2514 (1980).

[34] L. P. Kadanoff and P. C. Martin, Ann. Phys. (N.Y.) 24, 419 (1963).

[35] Because of the presence of a finite graviton mass the mechanical pressure, $\mathcal{P} \equiv T_{x x}$, and the thermodynamic pressure, $p \equiv-\Omega / \mathcal{V}$, are not equivalent [36].

[36] L. Alberte, M. Baggioli, and O. Pujolas, J. High Energy Phys. 07 (2016) 074.

[37] We have also checked the linear superpositions of the type $V(X)=\sum c_{\nu} X^{\nu}$ with $\nu>5 / 2$; the qualitative results remain unchanged.

[38] We fitted the data to $\omega=C_{1} k^{\alpha_{1}}+i C_{2} k^{\alpha_{2}}$. In all cases we found similar values: $\alpha_{1}=1.00001 \pm 4 \times 10^{-5}$ and $\alpha_{2}=2.00002 \pm 1 \times 10^{-5}$.

[39] S. A. Hartnoll, D. M. Ramirez, and J. E. Santos, J. High Energy Phys. 03 (2016) 170.

[40] J. C. Dyre, Rev. Mod. Phys. 78, 953 (2006).
[41] L. Berthier and G. Biroli, Rev. Mod. Phys. 83, 587 (2011).

[42] A. Cavagna, Phys. Rep. 476, 51 (2009).

[43] See Supplemental Material at http://link.aps.org/ supplemental/10.1103/PhysRevLett.120.171602 for the background solution, thermodynamics and the elasticity as well as perturbations and Green's functions, which includes Refs. [36,40-42,44,45].

[44] M. Baggioli and D. K. Brattan, Classical Quantum Gravity 34, 015008 (2017).

[45] M. Ammon, M. Kaminski, R. Koirala, J. Leiber, and J. Wu, J. High Energy Phys. 04 (2017) 067.

[46] R. A. Davison, B. Goutéraux, and S. A. Hartnoll, J. High Energy Phys. 10 (2015) 112.

[47] P. Burikham and N. Poovuttikul, Phys. Rev. D 94, 106001 (2016).

[48] T. Ciobanu and D. M. Ramirez, arXiv:1708.04997.

[49] L. V. Delacrétaz, B. Goutéraux, S. A. Hartnoll, and A. Karlsson, Phys. Rev. B 96, 195128 (2017).

[50] A. J. Beekman, J. Nissinen, K. Wu, K. Liu, R.-J. Slager, Z. Nussinov, V. Cvetkovic, and J. Zaanen, Phys. Rep. 683, 1 (2017).

[51] M. Micoulaut, Rep. Prog. Phys. 79, 066504 (2016).

[52] D. Anninos, T. Anous, F. Denef, and L. Peeters, J. High Energy Phys. 04 (2015) 027.

[53] M. Zacharias, I. Paul, and M. Garst, Phys. Rev. Lett. 115, 025703 (2015).

[54] M. Zacharias, A. Rosch, and M. Garst, Eur. Phys. J. Spec. Top. 224, 1021 (2015).

[55] L. V. Delacrétaz, B. Goutéraux, S. A. Hartnoll, and A. Karlsson, SciPost Phys. 3, 025 (2017).

[56] S. Grozdanov and N. Poovuttikul (to be published).

[57] A. Amoretti, D. Areán, B. Goutéraux, and D. Musso, Phys. Rev. D 97, 086017 (2018).

[58] A. Amoretti, D. Areán, B. Goutéraux, and D. Musso, following Letter, Phys. Rev. Lett. 120, 171603 (2018). 